This information is current as of April 26, 2023.

Fast Whole-Brain 4D Contrast-Enhanced MR Angiography with Velocity Encoding Using Undersampled Radial Acquisition and Highly Constrained Projection Reconstruction: Image-Quality Assessment in Volunteer Subjects

Y. Wu, W. Chang, K.M. Johnson, J. Velikina, H. Rowley, C. Mistretta and P. Turski

AJNR Am J Neuroradiol 2011, 32 (3) E47-E50

doi: https://doi.org/10.3174/ajnr.A2048

http://www.ajnr.org/content/32/3/E47 
TECHNICAL NOTE

\author{
Y. Wu \\ W. Chang \\ K.M. Johnson \\ J. Velikina \\ H. Rowley \\ C. Mistretta \\ P. Turski
}

\section{Fast Whole-Brain 4D Contrast-Enhanced MR Angiography with Velocity Encoding Using Undersampled Radial Acquisition and Highly Constrained Projection Reconstruction: Image- Quality Assessment in Volunteer Subjects}

\begin{abstract}
SUMMARY: We report on the image quality obtained by using fast contrast-enhanced whole-brain 4D radial MRA with 0.75 -second temporal resolution, isotropic submillimeter spatial resolution, and velocity encoding (HYPRFlow). Images generated by HYPR-LR by using the velocity-encoded data as the constraining image were of diagnostic quality. In addition, we demonstrate that measurements of shear stress within the middle cerebral artery can be derived from the high-resolution 3D velocity data.

ABBREVIATIONS: AV = arteriovenous; CE-VIPR = contrast-enhanced VIPR; HYPR = highly constrained back-projection; HYPRFlow = HYPR with phase contrast as constraint; HYPR-LR = HYPR local reconstruction; $\mathrm{MCA}=$ middle cerebral artery; $\mathrm{MIP}=$ maximum intensity projection; $\mathrm{MRA}=$ MR angiography; PC-VIPR = phase-contrast VIPR; SNR = signal intensity-to-noise ratio; VIPR = vastly undersampled isotropic projection reconstruction; WSS = wall shear stress
\end{abstract}

W hole-brain 4D contrast-enhanced MRA has been previously described by using Cartesian acquisitions, partialFourier encoding, and parallel imaging with multiple coils. ${ }^{1-3}$ However, performing high-resolution whole-brain 4D contrast-enhanced MRA examinations is challenging using conventional imaging methods because acquisition time increases proportionally with matrix size. Radial acquisitions like 2D projection reconstruction ${ }^{4-6}$ and $\mathrm{PC}-\mathrm{VIPR}^{7,8}$ can be effective in accelerating MR angiography; however, image quality is limited by undersampling artifacts and decreased SNR at high acceleration factors. Innovative reconstruction algorithms, such as HYPR and HYPR-LR ${ }^{9-11}$ ), which exploit prior information to constrain individual images, are able to reduce the severity of undersampling artifacts and SNR loss even at high levels of acceleration. Combining undersampled radial acquisition with HYPR reconstruction can dramatically improve frame rates and image coverage without degradation of image quality. ${ }^{12}$

HYPRFlow is one of the hybrid HYPR techniques that uses the phase-contrast image from a separate scan as the vascular constraint to provide whole-brain 4D contrast-enhanced MRA with subsecond temporal resolution and submillimeter isotropic spatial resolution as well as flow dynamics. ${ }^{12-14}$ In this article, we investigate the clinical utility of this technique by assessing the image quality of HYPRFlow and estimate WSS

Received August 20, 2009; accepted after revision December 14

From the Departments of Medical Physics (Y.W., W.C., J.V., C.M.), Radiology (W.C., H.R., C.M., P.T.), and Biomedical Engineering (K.M.J.), University of Wisconsin, Madison, Wisconsin; and University of Wisconsin School of Medicine and Public Health (W.C.), Madison, Wisconsin

P. Turski was supported by the National Institutes of Health grant R21 EB009441.

Please address correspondence to Yijing Wu, PhD, Department of Medical Physics, University of Wisconsin, Wisconsin Institutes for Medical Research, 1111 Highland Ave, Rm 1125, Madison, WI 53705; e-mail: yijingwu@wisc.edu

Indicates open access to non-subscribers at www.ajnr.org

DOI 10.3174/ajnr.A2048 in the middle cerebral arteries by using the time-average velocity through a single cardiac cycle.

\section{Technique}

Volunteer studies were performed in compliance with the Health Insurance Portability and Accountability Act regulations and by using a protocol approved by the local institutional review board. Eight adult subjects ranging from 24 to 40 years of age were imaged (4 women, 4 men) with a clinical 3T MR imaging system (Excite HD, TwinSpeed, Signa; GE Healthcare, Waukesha, Wisconsin) with an 8-channel head coil (HD Brain Coil, GE Healthcare). Each HYPRFlow study involved 2 encoding methods. First, during contrast injection, spatial encoding was performed by using a time-resolved multiecho 3D radial acquisition (CE-VIPR). ${ }^{15}$ Subsequently, velocity encoding was performed by using a high-resolution dual-echo $3 \mathrm{D}$ radial phase-contrast acquisition (PC-VIPR). The PC-VIPR data were used to form a composite image (angiographic constraint) for HYPR-LR reconstruction and for hemodynamic evaluation.

\section{MR Imaging Protocol}

Imaging parameters for CE-VIPR were the following: $\mathrm{FOV}=26 \times 26 \times$ $26 \mathrm{~cm}^{3}, \mathrm{TR} / \mathrm{TE}=3.0 / 0.4 \mathrm{~ms}$, bandwidth $=125 \mathrm{kHz}, 64$ points from the center to the edge of the $k$-space for each projection, frame update time $=$ 0.5 seconds. Scanning parameters for postcontrast PC-VIPR were the following: $\mathrm{FOV}=22 \times 22 \times 22 \mathrm{~cm}^{3}$, TR/TE $=12.5 / 4.8 \mathrm{~ms}$, velocity encoding $=60 \mathrm{~cm} / \mathrm{s}$, bandwidth $=62.5 \mathrm{kHz}$. The readout matrix was 320 points per projection, and the spatial resolution for the composite image was $0.7 \times 0.7 \times 0.7 \mathrm{~mm}^{3}$. Seven thousand projections were acquired within 5 minutes. Gadobenate dimeglumine (MultiHance, Bracco Diagnostics, Princeton, New Jersey) was injected at $3 \mathrm{~mL} / \mathrm{s}$, and the contrast dose was $0.1 \mathrm{~mm} / \mathrm{kg}$ followed by a $20-\mathrm{mL}$ saline flush.

\section{Image Reconstruction}

In the HYPRFlow method, complex difference images were reconstructed from the postcontrast PC-VIPR (spatial resolution $=0.7 \times$ $0.7 \times 0.7 \mathrm{~mm}^{3}$ ) and were used as a composite for the HYPR-LR algorithm. The temporal weighting images were used to define the contrast 

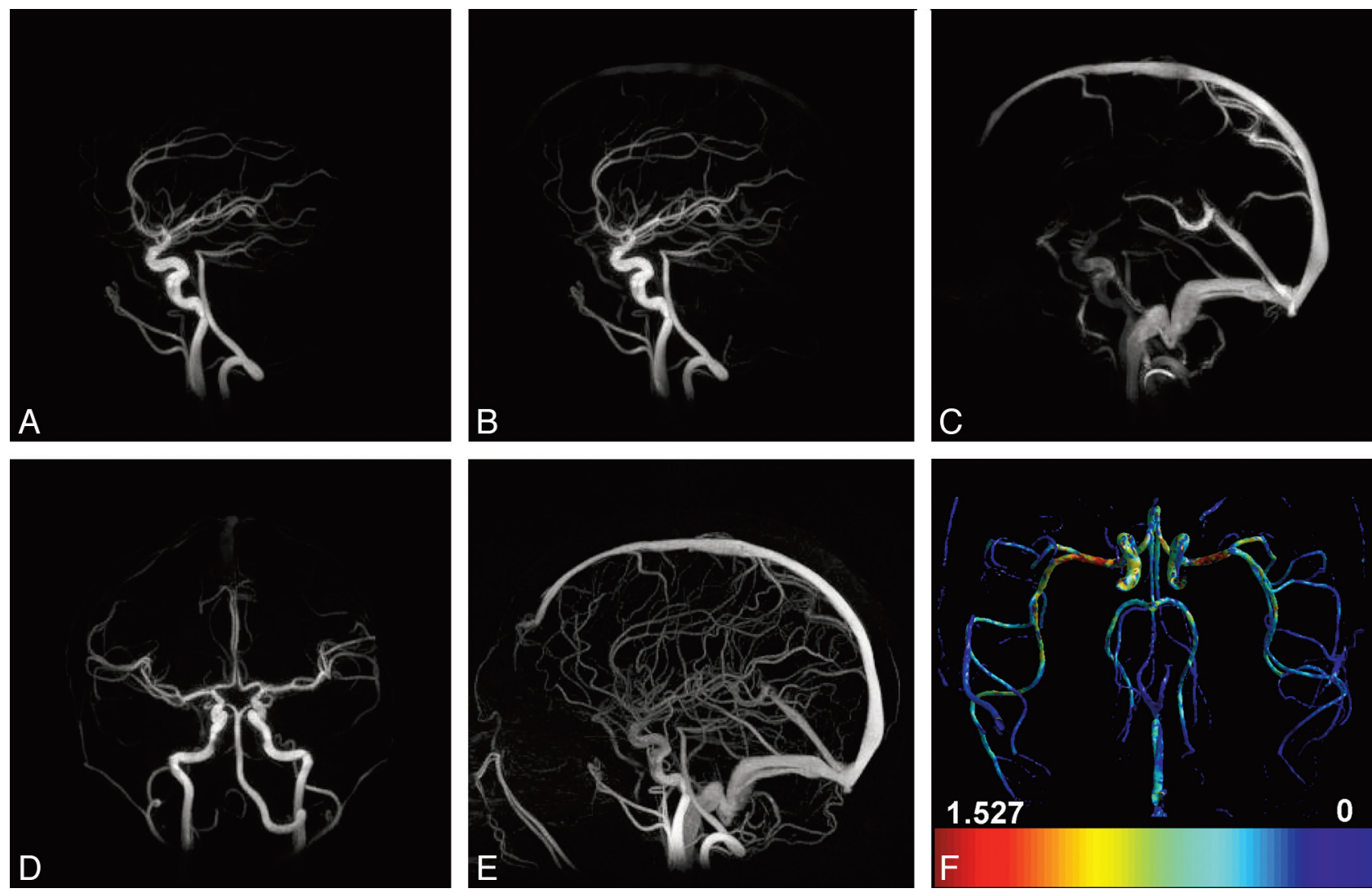

Fig 1. $A$, Sagittal HYPRFlow timeframe in the early arterial phase. $B$, Sagittal HYPRFlow timeframe in the late arterial phase. $C$, Sagittal HYPRFlow timeframe in the venous phase. $D$, Coronal HYPRFlow timeframe in the arterial phase. E, Sagittal PC-VIPR MIP. F, WSS map of the axial whole brain generated from PC-VIPR velocity data.

kinetics obtained by convolving timeframe images (reconstructed from the CE-VIPR) with a locally averaging kernel. ${ }^{8}$ Adaptive $k$-space filtering with a 0.75 -second temporal window at the cutoff frequency was used to suppress the high-spatial-frequency undersampling artifacts and increase the SNR while maintaining a high temporal resolution. ${ }^{16}$ Following HYPR-LR reconstruction, the result was a time series of contrastenhanced MRAs with both high temporal resolution ( 0.75 seconds) and high spatial resolution $\left(0.7 \times 0.7 \times 0.7 \mathrm{~mm}^{3}\right)$.

\section{SNR Comparison}

To quantitatively compare the SNR improvement of HYPRFlow, we drew, for each volunteer, 3 target regions of interest from the internal carotid artery, basilar artery, and sinus veins at peak arterial and peak venous phases. Corresponding background regions of interest were drawn near the target regions of interest at the same timeframes. SNRs were measured from HYPRFlow images and CE-VIPR images.

\section{Image-Quality Analysis}

HYPRFlow arterial and venous phase images were scored by 2 experienced observers for image quality in the carotid siphons, A1, M1, P1, basilar artery, deep veins, and dural sinuses. Images were scored on a scale of 1-4 (1, not visible; 2 , nondiagnostic; 3 , diagnostic; 4, well-delineated). The examinations were graded for temporal image quality by scoring AV separation (1, no AV separation; 2, AV separation but vessels obscured by overlap; 3, AV separation adequate for diagnostic purposes; 4, excellent AV separation with minimal arterial signal intensity).

\section{Results}

The top row of Figure 1 shows 3 representative HYPRFlow timeframes (out of 120 timeframes in total) demonstrating early arterial phase (Fig $1 A$ ), late arterial phase (Figure $1 B$ ), and late late venous phase (Fig $1 C$ ) in sagittal view. Figure $1 D$ shows the late arterial phase in coronal plane. Figure $1 E$ is the sagittal MIP of the complex different images acquired from the phase contrast scan and served as the spatial constraint for the HYPRFlow reconstruction. Figure $1 F$ shows a map of estimated WSS derived from the velocity data. Average HYPRFlow spatial image quality scores were 3.50 for the arterial phase and 3.81 for the venous phase, and AV separation was scored as 3.56. PC-VIPR image quality was 3.39 for arteries and 3.78 for venous structures. The difference in image quality between HYPRFlow and PC-VIPR was not statistically significant $(P=.1075$, Wilcoxon rank sum test). Interobserver $\kappa$ analysis showed $78.6 \%$ concordance with $\kappa=0.714$, which is considered good agreement. The interobserver error for observers 1 and 2 was $3.8 \%$ (SD differences, $13.3 \%-18.4 \%$ ). The average SNR measurements were $50.5 \pm 19.5$ for HYPRFlow images and $22.6 \pm 13.1$ for CE-VIPR images. The difference was statistically significant $(P<.0001)$. WSS values measured in the proximal M1 segment ranged from 0.8 to $1.6 \mathrm{~N} / \mathrm{m}^{2}$, and there was no significant difference in WSS between the right and left M1 segments of the MCA $(P=.44)$.

\section{Discussion}

Previous methods of whole-brain time-resolved contrastenhanced MRA using Cartesian acquisitions were limited by long scanning times, relatively low spatial resolution from few phase encodes, or low temporal resolution due to view sharing. Parallel imaging techniques such as sensitivity encoding allow acceleration via unfolding of $k$-space (at the expense of 
SNR), but the acceleration factor is limited to the number of coils ( 8 in standard head coils) and noise at that acceleration factor would be prohibitive. ${ }^{17,18}$ HYPRFlow avoids these limitations by using 2 separate scans to achieve high temporal and spatial resolution and by exploiting the sparsity inherent in brain MRA to achieve high acceleration factors while maintaining an acceptable SNR.

Phase-contrast radial acquisition is particularly well-suited to cerebrovascular imaging because most tissue in the FOV is stationary and is subtracted during reconstruction, leading to enhanced sparsity, which allows a high level of undersampling because streak artifacts are diffuse, reduced, and projected outside the region of interest at the center of image space. The contrast-enhanced time-resolved image is also sparse because a mask is acquired before the contrast injection and then subtracted, with only the areas with contrast left. Thus, the contrast-enhanced time-resolved acquisition and the phase-contrast data used as the composite are sparse; this feature allows HYPR-LR to provide near-exact reconstruction even at high levels of acceleration. ${ }^{8}$

Shear stress is an important physiologic parameter, which is associated with the development of atherosclerotic plaque. ${ }^{19}$ Prior studies of estimated WSS by using phase-contrast techniques were limited to 2D sections or very time-consuming 3D phase-contrast acquisitions. Prior reports have shown a high degree of correlation between PC-VIPR and catheter-based endovascular measurements of physiologic parameters. ${ }^{20,21}$ In our investigations, relatively high-resolution HYPRFlow velocity data were used to estimate WSS in the proximal middle cerebral artery. We recognize that MR imaging underestimates WSS compared with techniques such as computational fluid dynamics ${ }^{22}$ because of insufficient resolution at the boundary and could yield slightly different results compared with other MR imaging WSS techniques, such as spline interpolation; we plan to use estimated WSS as a surrogate parameter to distinguish areas of high and low WSS.

Because HYPRFlow requires 2 acquisition modes (dynamic and velocity encoding), patient motion is a concern. However, when the subject moves between modes, image registration can be used before HYPR-LR reconstruction to reduce this problem. Another challenge is that the phase-contrast images are sensitive to flow. In the regions of complex and/or slow flow, phase-contrast MRA images may not sufficiently delineate the vasculature, causing diagnostically obscuring noise amplification in the final HYPRFlow images. Magnitude images from phase-contrast MRA, which are effectively postcontrast time-of-flight images, provide better depiction of the vasculature but are substantially less sparse; these features make HYPR processing more difficult. Further work is required to investigate alternative reconstruction schemes, such as iterative HYPR and compressed sensing techniques, ${ }^{23-26}$ which may provide better decoupling of the constraining image and data being reconstructed. This should allow the use of a composite that is a weighted combination of angiographic and magnitude images from postcontrast PC-VIPR.

Future research will include automated reconstruction software, investigating the use of HYPRFlow velocity data to generate pressure fields, velocity vector plots, streamline flow, and tracing particle paths through the flow field. Development of dual-velocity encoded methods is currently underway.

\section{Appendix}

\section{WSS Estimation}

To measure WSS, we accept that the achievable temporal and spatial resolution with MR imaging is not sufficient to depict the vessel boundary layer. However, we aim to use MR imaging-derived WSS as a surrogate parameter that can be used to compare the heterogeneous WSS distribution. For blood, the WSS can be approximated as

$$
W S S=-\left.\mu \frac{\partial V_{T}}{\partial \hat{n}}\right|_{\text {wall }},
$$

where $\mu$ is the viscosity (assumed to be $4.0 \mathrm{cP}$ ), $\mathrm{V}_{T}$ is the velocity tangent to the wall, and $\hat{n}$ is the normal vector of the wall. The algorithm to compute 3D WSS has 3 basic steps: 1) segmentation of the vessel boundaries, 2) calculation of the normal vector, and 3) velocity fitting. ${ }^{27,28}$ Segmentation used an isosurface of the angiographic data with a manually chosen value as the minimum value that did not introduce significant noise. Image normal vectors were determined by using the gradient of the angiogram at the segmented vessel wall locations. Second-order polynomials were used to fit the velocity data and determine the velocity derivative. An average WSS map was generated by taking the average velocity through a cardiac cycle. Small segments $(\sim 100$ voxels inside each segment, corresponding to $35-\mathrm{mm}^{3}$ volume) in the middle of the MCAs were selected to represent the WSS estimation of the MCA.

\section{References}

1. Willinek W, Hadizadeh, D. Falkenhausen, et al. 4D time-resolved MR angiography with keyhole (4D-TRAK): more than 60 times accelerated MRA using a combination of CENTRA, keyhole, and SENSE at 3.0T. J Magn Reson Imaging 2008;27:1455-60

2. Fink C, Ley S, Kroeker R, et al. Time-resolved contrast-enhanced three-dimensional magnetic resonance angiography of the chest: combination of parallel imaging with view sharing (TREAT). Invest Radiol 2005;40:40-48

3. Parmar H, Ivancevic M, Dudek, et al. Neuroradiologic applications of dynamic MR angiography at 3T. Magn Reson Imaging Clin N Am 2009;17:63-75

4. Peters D, Korosec F, Grist T, et al. Undersampled projection reconstruction applied to MR angiography. Magn Reson Med 2000;43:91-101

5. Barger A, Bloch W, Toropov Y, et al. Time-resolved contrast-enhanced imaging with isotropic resolution and broad coverage using an undersampled $3 \mathrm{D}$ projection trajectory. Magn Reson Med 2002:48:297-305

6. Lu A, Wieben $\mathrm{O}$, Grist $\mathrm{T}$, et al. Vastly undersampled isotropic projection reconstruction imaging with multi-half-echo (VIPR ME). In: Proceedings of the 11th Annual Meeting of International Society of Magnetic Resonance in Medicine, Toronto, Ontario, Canada. July 10-16, 2003;50

7. Gu T, Korosec F, Block W, et al. PC VIPR: a high-speed 3D phase-contrast method for flow quantification and high-resolution angiography. AJNR Am J Neuroradiol 2005;26:743-49

8. Johnson K, Lum D, Turski P, et al. Improved 3D phase contrast MRI with off-resonance corrected dual echo VIPR. Magn Reson Med 2008;60:1329-36

9. Mistretta C, Wieben O, Velikina J, et al. Highly constrained backprojection for time-resolved MRI. Magn Reson Med 2006;55:30-40

10. Johnson $\mathrm{K}$, Velikina $\mathrm{J}$, $\mathrm{Wu} \mathrm{Y}$, et al. Improved waveform fidelity using local HYPR reconstruction (HYPR LR). Magn Reson Med 2008;59:456-62

11. Supanich M, Tao Y, Nett B, et al. Radiation dose reduction in time-resolved CT angiography using highly constrained back projection reconstruction. Phys Med Biol 2009;54:4575-93

12. Mistretta C. Undersampled radial MR acquisition and highly constrained back projection (HYPR) reconstruction: potential medical imaging applications in the post-Nyquist era. J Magn Reson Imaging 2009;29:501-16

13. Velikina J, Johnson K, Wieben O, et al. PC HYPRFlow: a technique for rapid imaging of contrast dynamics. J Magn Reson Imaging 2010;37:447-56

14. Wu Y, Johnson K, Velikina J, et al. Clinical experience of HYPR Flow. In: 
Proceedings of the 16th Annual Meeting of International Society of Magnetic Resonance in Medicine, Toronto, Ontario, Canada. May 3-9, 2008;20

15. Brodsky EK, Lu A, Thornton FJ, et al. Using multiple half-echoes to improve sampling efficiency and fat suppression in time-resolved MRA. In: Proceedings of the 11th Annual Meeting of International Society of Magnetic Resonance in Medicine, Toronto, Ontario, Canada. July 10-16, 2003;24

16. Du J, Carroll T, Brodsky E, et al. Contrast-enhanced peripheral magnetic resonance angiography using time-resolved vastly undersampled isotropic projection reconstruction. J Magn Reson Imaging 2004;20:894-900

17. Golay X, Brown S, Itoh R, et al. Time-resolved contrast-enhanced carotid MR angiography using sensitivity encoding (SENSE). AJNR Am J Neuroradiol 2001;22:1615-19

18. Glockner JF, Hu HH, Stanley DW, et al. Parallel MR imaging: a user's guide. Radiographics 2005;25:1279-97

19. Melek AM, Alpers SI, Izumo S. Hemodynamic shear stress and its role in atherosclerosis. JAMA 1999;282:2035-42

20. Turk AS, Johnson K, Lum D, et al. Physiologic and anatomic assessment of a canine carotid artery stenosis model utilizing phase contrast with vastly undersampled isotropic projection imaging. AJNR Am J Neuroradiol 2007; 28:111-15

21. Moftakhar R, Aagaard-Kienitz B, Johnson K, et al. Noninvasive measurement of intra-aneurysmal pressure and flow pattern using phase contrast with vastly undersampled isotropic projection imaging. AJNR Am J Neuroradiol 2008;28:1710-14

22. Wood N, Weston S, Kilner P, et al. Combined MR imaging and CFD simulation of flow in the human descending aorta. J Magn Reson Imaging 2001;13:699-713

23. Velikina J, Samsonov A. HYPR- 10: a hybrid technique for CE-MRA with extreme undersampling factors. Presented at: International Society of Magnetic Resonance in Medicine Workshop on Data Sampling and Image Reconstruction, Sedona, Arizona, January 25-28, 2009

24. O'Halloran RL, Wen Z, Holmes JH, et al. Iterative projection reconstruction of time-resolved images using highly-constrained back-projection (HYPR). Magn Reson Med 2008;59:132-39

25. Griswold M, Barkauskas K, Blaimer M, et al. More optimal HYPR reconstructions using a combination of hyper- and conjugate-gradient minimization. In: Proceedings of the International Society of Magnetic Resonance in Medicine, Berlin, Germany. May 19-25, 2007;188

26. Lustig M, Donoho D, Pauly JM. Sparse MRI: the application of compressed sensing for rapid MR imaging. Magn Reson Med 2007;58:1182-95

27. Papathanasopoulou P, Zhao S, Kohler U, et al. MRI measurement of timeresolved wall shear stress vectors in a carotid bifurcation model, and comparison with CFD predictions. J Magn Reson Imaging 2002;17:153-62

28. Oshinski JN, Ku DN, Mukundan S Jr, et al. Determination of wall shear stress in the aorta with the use of MR phase velocity mapping. J Magn Reson Imaging 1995;5:640-47 